\title{
Lymph Node Ratio: Is It an Independent Prognostic Factor for Stage III Cutaneous Melanoma?
}

\author{
Abolfazl Salari ${ }^{1}$, Fatemeh Nili ${ }^{2}$, Amir Mohsen Jalaeefar ${ }^{3}$, Mohammad \\ Shirkhoda ${ }^{1,3 *}$
}

\begin{abstract}
Objective: Lymph node ratio (LNR) is defined as the ratio of the number of metastatic lymph nodes to the dissected lymph nodes. LNR is a prognostic factor for many tumor types. The present study aimed to evaluate the prognostic value of LNR in melanoma. Methods: This retrospective cohort study was conducted on 123 patients with stage III cutaneous melanoma. Multivariate Cox proportional hazards model was used to evaluate the correlations between LNR and other clinicopathological factors associated with survival. The patients were divided into four groups in terms of the LNR, including groups $\mathrm{A}(\mathrm{LNR} \leq 0.18), \mathrm{B}(0.18<\mathrm{LNR} \leq 0.37), \mathrm{C}(0.37<\mathrm{LNR} \leq 0.625)$, and $\mathrm{D}(\mathrm{LNR}>0.625)$. Results: Initially, LNR was evaluated as a continuous quantity associated with survival. In the univariate analysis, a significant correlation was observed between LNR, overall survival (OS), and disease free survival (DFS). Meanwhile, the only association observed in the multivariate analysis was between LNR and OS. Increased LNR from group A to group D reduced OS from $46( \pm 44.09)$ to $22.5( \pm 16.33)$ months $(\mathrm{P}=0.022)$. According to the multivariate analysis, prognostic factors in OS were tumor thickness, American joint committee of cancer (AJCC) N stage, interferon administration, and undergoing chemotherapy. Conclusion: According to the results, LNR could be used as an independent prognostic factor for estimating the survival of patients with stage III cutaneous melanoma also designing an effective adjuvant treatment protocol for these patients.
\end{abstract}

Keywords: Cutaneous melanoma- lymph node ratio- lymph node dissection

Asian Pac J Cancer Prev, 19 (12), 3623-3627

\section{Introduction}

Similar to other cancers, some of the factors involved in cutaneous melanoma could estimate the severity of the disease and survival of the patients. In melanoma, pathological factors, such as Breslow thickness and primary ulcerated tumors, are influential in the prognosis of the patients. In addition, the number of metastatic lymph nodes $(\mathrm{N})$ is considered to be the most powerful determinant of the survival of patients with stage III melanoma (Balch et al., 2010).

Evidence suggests that survival rate decreases from $87 \%$ to $23 \%$ in the patients with one micro-metastasis in the lymph nodes of non-ulcerated primary tumors with the thickness of less than 2 millimeters compared to the patients with four or more macro-metastases in the lymph nodes of ulcerated primary tumors with the thickness of more than 6 millimeters. Although the survival rate decreases with the increased thickness and ulceration of the tumor, the impact of these variables is not as significant as the number of the metastatic lymph nodes on the reduction of survival rate in the patients with melanoma (Balch et al., 2010). Considering the key role on the determinants of prognostic factors in the need for adjuvant therapy in the patients (Eggermont et al., 2008), estimating the number of metastatic lymph nodes is of paramount importance in this regard.

The current treatment for the melanoma patients with metastatic lymph nodes involvement is complete lymph node dissection (CLND). However, only $50 \%$ of these have been reported to undergo CLND (Bilimoria et al., 2008). The number of the lymph nodes that must be dissected as CLND in each lymphatic region in melanoma remains unclear. Inadequate dissection leads to the reduced number of positively dissected lymph nodes, which is effective in determining the stage of the disease, prognosis of the patients, and selection the treatment. Surgeons in various medical centers have different capabilities and experiences in lymph nodes dissection (Spillane et al., 2009). Therefore, lymph node ratio (LNR) has been proposed as a new factor to diminish the effects of inadequate lymph node dissection. LNR is defined as the ratio of the number of the positive lymph nodes to the total number of the removed lymph nodes. 
According to recent studies, LNR acts as an independent factor for estimating the survival of patients with melanoma (Rossi et al., 2008; Xing et al., 2009), as well as those with other cancer types, including colorectal (Berger et al., 2005; Wang et al., 2008; Zhou et al., 2015), gastrointestinal (Bhamidipati et al., 2012; Zhang et al., 2013; Zhou et al., 2013), breast (Truong et al., 2008), periampullary (Farid et al., 2014), and pancreatic cancer (Yamamoto et al., 2014; Zhan et al., 2015) and head and neck squamous cell carcinoma (Sano et al., 2018).

With this background in mind, the present study aimed to evaluate the role of LNR in the prognosis of patients with stage III melanoma. The main question raised in the current research was whether LNR acts as an independent prognostic factor for determining the survival rate in these patients.

\section{Materials and Methods}

\section{Patients}

This retrospective cohort study was conducted on the patients with the pathologic diagnosis of cutaneous melanoma, who were admitted to the Cancer Institute at Imam Khomeini Hospital complex, affiliated to Tehran University of Medical Sciences, Tehran, Iran during 2003-2015. Inclusion criteria of the study were the diagnosis of cutaneous melanoma, undergoing lymph node dissection, and a minimum of one metastatic dissected lymph node.

Exclusion criteria of the study were as follows: 1) history of cancer; 2) presence of other skin cancers; 3) distant metastases since the diagnosis; 4) involvement of more than one lymphatic region (e.g., patients with a trunk melanoma that metastasized to axillary and inguinal lymph nodes) and 5) lack of access for follow-up.

\section{Data Collection}

Demographic and histological characteristics, number of the involved lymph nodes, and dissected lymph nodes of the patients were collected by reviewing their medical records. To calculate LNR, the number of the metastatic lymph nodes was divided by the total dissected lymph nodes. Moreover, data on recurrence and death of the patients were obtained via telephone interviews.

\section{Statistical Analysis}

Qualitative variables were expressed as number and percentage, and quantitative variables were expressed as mean and standard deviation. Death of the patients was considered the 'main event' in the survival analysis, and semi-parametric Cox proportional hazards models were used at this stage. Proportional hazards assumption was evaluated for all the variables affecting the survival of the patients. In addition, the effects of confounding and underlying variables were modified in the statistical modeling.

Survival function was estimated using the Kaplan-Meier estimator, and goodness of fit of the model was assessed using the Akaike's information criterion (AIC). The quantile of the quantitative LNR values was used to categorize the variable (first quantile $=0.18$, second quantile $=0.37$, third quantile $=0.625$ ). Data analysis was performed in STATA version 13, and P-value of less than 0.05 was considered significant.

\section{Results}

Clinicopathological data of the patients undergoing lymph node dissection $(\mathrm{N}=123)$ are presented in Table 1. Mean follow-up of the patients was 45.24 $( \pm 38.48)$ months. The five-year survival rate of the patients was $34.1 \%$, and 89 patients $(72.4 \%)$ died due to melanoma. According to the results, the mean number of the dissected and metastatic lymph nodes was 14.57 $( \pm 11.14)$ and $5.76( \pm 7.1)$, respectively. In addition, $49.2 \%$ of the patients had a minimum of 4 metastatic lymph nodes (AJCC N3 stage).

Initially, LNR was evaluated as a continuous variable in order to assess its association with the survival of the patients. In the univariate analysis, a significant correlation was observed between LNR and disease free survival (DFS), as well as LNR and overall survival (OS) (Table 2). However, in the multivariate analysis, LNR failed to anticipate DFS (OS: $\mathrm{HR}=48.18$; 95\% CI: 3.24-716.2; $\mathrm{P}=0.005$; DFS: $\mathrm{HR}=5.82,95 \% \mathrm{CI}: 0.54-62.55 ; \mathrm{P}=0.15$ ).

Patients were classified into four groups in terms of the LNR, including groups $\mathrm{A}(\mathrm{LNR} \leq 0.18)$, B $(0.18<\mathrm{LNR} \leq 0.37), \mathrm{C}(0.37<\mathrm{LNR} \leq 0.625)$, and $\mathrm{D}$ $(\mathrm{LNR}>0.625)$. With the increased LNR from group A to group D, mean OS decreased from $46( \pm 44.09)$ to 22.5 $( \pm 16.33)$ months $(\mathrm{P}=0.022)$ (Table 3; Figures 1 and 2$)$. Moreover, AIC was applied to compare the accuracy of the AJCC (American joint committee of cancer) N stage and LNR models in the anticipation of OS. The AIC score for LNR was 197, while it was 215 for the AJCC N Stage. The lower number of AIC indicated the accuracy of the model.

In the multivariate analysis of the variables, OS was observed to be significantly higher in the patients receiving interferon and chemotherapy. Furthermore, the mortality rate was three times higher in the patients with N3 compared to those with N1 (P-value: 0.04), while the mortality rate was 6.6 times higher in the patients who experienced increased primary tumor thickness from $\mathrm{T} 1$

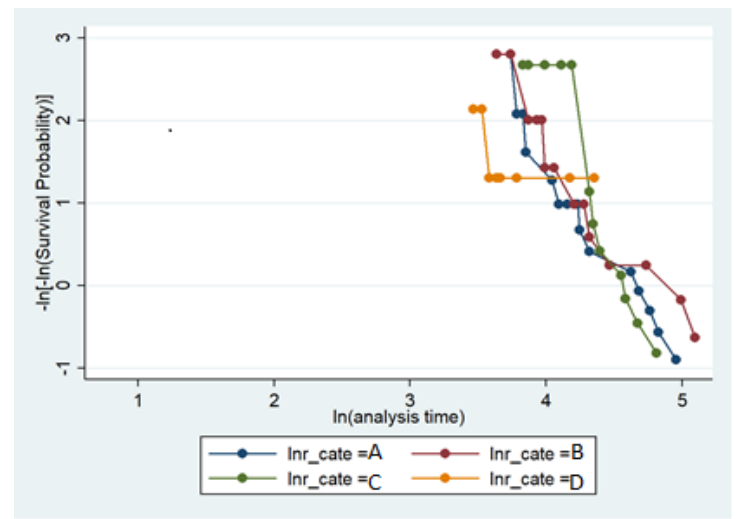

Figure 1. Comparison of Overall Survival Time between LNR Categories (Cate. A, LNR $\leq 0.18$; Cate. $\mathrm{B}, 0.18<\mathrm{LNR} \leq 0.37$; Cate. $\mathrm{C}, 0.37<\mathrm{LNR} \leq 0.625$; Cate. D, LNR $>0.625)$, PH Graphical Assessment. 
Table 1. Participants' Characteristics

\begin{tabular}{|c|c|}
\hline Characteristics & $\begin{array}{c}\text { Descriptive } \\
\text { Statistics }(\mathrm{N}=12\end{array}$ \\
\hline Age (Year)* & $56.30 \pm 14.53$ \\
\hline \multicolumn{2}{|l|}{ Sex } \\
\hline Male & $80(65 \%)$ \\
\hline Female & - \\
\hline \multicolumn{2}{|l|}{ Ulcerate } \\
\hline Yes & $65(73.9 \%)$ \\
\hline No & - \\
\hline \multicolumn{2}{|l|}{ IFN } \\
\hline Yes & $85(88.5 \%)$ \\
\hline No & - \\
\hline \multicolumn{2}{|l|}{ Chemotherapy } \\
\hline Yes & $80(68.4 \%)$ \\
\hline No & - \\
\hline \multicolumn{2}{|l|}{ Radiotherapy } \\
\hline Yes & $69(59.5 \%)$ \\
\hline No & - \\
\hline \multicolumn{2}{|l|}{ Filed of melanoma } \\
\hline Head and neck & $22(17.9 \%)$ \\
\hline Trunk & $9(7.3 \%)$ \\
\hline Extremities & - \\
\hline \multicolumn{2}{|l|}{ Type of melanoma } \\
\hline SS & $11(12.2 \%)$ \\
\hline $\mathrm{AL}$ & $29(32.2 \%)$ \\
\hline NODULAR & $42(46.7 \%)$ \\
\hline $\mathrm{LM}$ & - \\
\hline \multicolumn{2}{|l|}{ LN.F } \\
\hline Axillary & $29(23.6 \%)$ \\
\hline head and neck & $22(17.9 \%)$ \\
\hline Ilioinguinal & - \\
\hline \multicolumn{2}{|l|}{$\mathrm{N}$} \\
\hline N1 & $26(21.3 \%)$ \\
\hline N2 & $36(29.5 \%)$ \\
\hline N3 & $60(49.2 \%)$ \\
\hline \multicolumn{2}{|l|}{$\mathrm{T}$} \\
\hline $\mathrm{T} 1$ & $10(8 \%)$ \\
\hline $\mathrm{T} 2$ & $23(19 \%)$ \\
\hline $\mathrm{T} 3$ & $28(23 \%)$ \\
\hline $\mathrm{T} 4$ & $62(50 \%)$ \\
\hline \multicolumn{2}{|l|}{ LNR } \\
\hline $\mathrm{LNR} \leq 0.18$ & $29(23.6 \%)$ \\
\hline $0.18<\mathrm{LNR} \leq 0.37$ & $31(25.2 \%)$ \\
\hline $0.37<\mathrm{LNR} \leq 0.625$ & $33(26.8 \%)$ \\
\hline $\mathrm{LNR}>0.625$ & $30(24.4 \%)$ \\
\hline Overall Survival (month) & $45.24 \pm 38.48$ \\
\hline Disease Free Survival (month) & $25.21 \pm 232.98$ \\
\hline LNR & $0.428 \pm 0.290$ \\
\hline
\end{tabular}

SS, Superficial Spreading; LM, Lentigo Maligna; AL, Acral Lentiginous, LNF: Lymph Node Field; IFN, Interferon; LNR, Lymph Node Ratio.

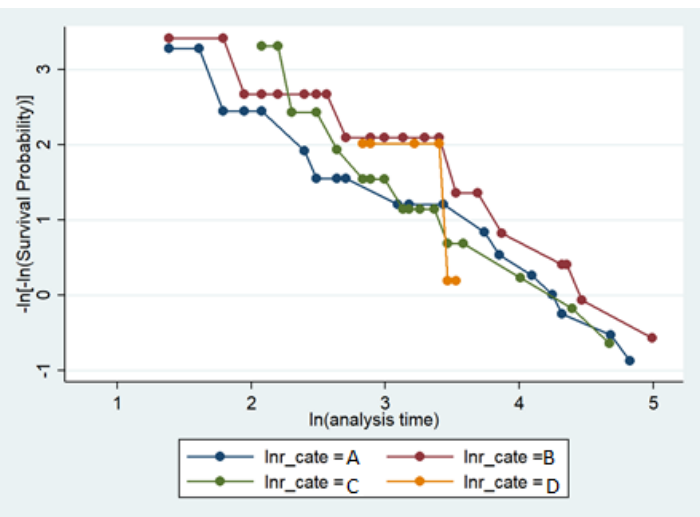

Figure 2. Comparison of Disease Free Survival Time between LNR Categories (Cate.A, LNR $\leq 0.18$; Cate.B, $0.18<\mathrm{LNR} \leq 0.37$; Cate. $\mathrm{C}, 0.37<\mathrm{LNR} \leq 0.625$; Cate. D, LNR $>0.625$ ), PH Graphical Assessment.

to T4 (P-value: 0.01) (Table 4). Nevertheless, the other primary characteristics of melanoma (e.g., primary site, ulceration, involved lymphatic region, and type of melanoma) had no impact on the OS of the patients.

\section{Discussion}

Prognostic factors could be used to identify high-risk patients at the stage IIIof melanoma in order to determine the adjuvant treatment protocol (Coit et al., 2016). In this regard, Balch et al. conducted a research on 2,313 melanoma patients with metastatic lymph nodes in 2010. According to the results, while age, ulceration, tumor thickness, nodal tumor burden, and the number of metastatic lymph nodes (AJCC N stage) affected the OS of the patients, the number of metastatic lymph nodes had the most significant impact on this parameter (Balch et al., 2010).

Although it is necessary that all melanoma patients with metastatic lymph nodes undergo CLND; valid oncology guidelines, such as those proposed by the European Society for Medical Oncology (ESMO) (Dummer et al., 2015) and the American Society of Clinical Oncology (ASCO) (Wong et al., 2018), have refused to present a specific number as the criterion for the minimum number of removed lymph nodes. In addition, several studies have suggested variable rates for the minimum number of dissected lymph nodes (Grotz et al., 2013; Sandro et al., 2015).

Considering the controversy over the number of dissected lymph nodes and differences in the skills of surgeons in the dissection of lymph nodes, lack of CLND and underestimation of the $\mathrm{N}$ stage in the patients become more likely. To address this issue, Rossi et al., (2008) evaluated the role of LNR as a new prognostic factor in the survival of melanoma patients. The mentioned research was conducted on 213 patients undergoing radical lymph node dissection, who were classified into three groups in terms of the LNR, including groups $\mathrm{A}(\mathrm{LNR} \leq 0.1), \mathrm{B}$ $(0.1<\mathrm{LNR} \leq 0.25)$, and $\mathrm{C}(\mathrm{LNR}>0.25)$. In the multivariate analysis, a significant correlation was reported between LNR and OS, whereas no significant association was observed between AJCC N stage and survival. 
Table 2. Assessing Effect of LNR on Overall Survival and Disease Free Survival Time by Univariate COX PH Model

\begin{tabular}{lccccccc}
\hline \multicolumn{3}{c}{ Overall Survival Time } & \multicolumn{5}{c}{ Free Disease Survival Time } \\
\hline Characteristics & HR & {$[95 \% \mathrm{CI}]$} & P-value & Characteristics & HR & {$[95 \%$ CI $]$} & P-value \\
LNR & 3.30 & {$[1.64-6.62]$} & 0.001 & LNR & 2.81 & {$[1.39-5.66]$} & 0.004 \\
\hline HR, Hazard Ratio; LNR, Lymph Node Ratio & & & &
\end{tabular}

Table 3. Comparison of Overall Survival and Disease Free Survival Time between the Categories of LNR (Median*土 SD for Survival Time Variables)

\begin{tabular}{lccccc}
\hline Characteristics & LNR $\leq 0.18$ & $0.18<\mathrm{LNR} \leq 0.37$ & $0.37<\mathrm{LNR} \leq 0.625$ & $\mathrm{LNR}>0.625$ & P-value \\
\hline Overall Survival & $46 \pm 44.09$ & $38 \pm 43.66$ & $28 \pm 37.76$ & $22.5 \pm 16.33$ & 0.022 \\
Disease Free Survival & $14 \pm 41.76$ & $18 \pm 40.22$ & $14 \pm 28.37$ & $12 \pm 8.42$ & 0.143 \\
\hline
\end{tabular}

*months

Table 4. Assessing the Effect of Study's Variables on Overall Survival Time by Multivariate COX PH Model

\begin{tabular}{|c|c|c|c|c|}
\hline Variable & & HR & {$[95 \% \mathrm{CI}]$} & P-value \\
\hline \multirow[t]{2}{*}{ Ulcerate } & Yes & 1.001 & {$[0.413,2.42]$} & 0.998 \\
\hline & No & Ref. & Ref. & Ref. \\
\hline \multirow[t]{2}{*}{ IFN } & Yes & 0.003 & {$[0.001,0.272]$} & 0.011 \\
\hline & No & Ref. & Ref. & Ref. \\
\hline \multirow[t]{3}{*}{ Chemotherapy } & Yes & 0.009 & {$[0.001,0.298]$} & 0.007 \\
\hline & No & Ref. & Ref. & Ref. \\
\hline & N1 & Ref. & Ref. & Ref. \\
\hline \multirow[t]{4}{*}{$\mathrm{N}$} & N2 & 1.50 & {$[0.245-9.27]$} & 0.66 \\
\hline & N3 & 2.9 & [1.15-11.19] & 0.04 \\
\hline & $\mathrm{T} 1$ & Ref. & Ref. & Ref. \\
\hline & $\mathrm{T} 2$ & 4.355 & {$[0.985,19.25]$} & 0.052 \\
\hline \multirow[t]{3}{*}{$\mathrm{T}$} & $\mathrm{T} 3$ & 5.925 & {$[1.37,25.52]$} & 0.017 \\
\hline & $\mathrm{T} 4$ & 6.638 & {$[1.59,27.76]$} & 0.010 \\
\hline & $\mathrm{LNR} \leq 0.18$ & Ref. & Ref. & Ref. \\
\hline \multirow[t]{3}{*}{ LNR } & $0.18<\mathrm{LNR} \leq 0.37$ & 0.005 & {$[0.001,0.35]$} & 0.015 \\
\hline & $0.37<\mathrm{LNR} \leq 0.625$ & 0.035 & {$[0.001,2.19]$} & 0.112 \\
\hline & $\mathrm{LNR}>0.625$ & 6.33 & {$[0.28,143]$} & 0.246 \\
\hline LNR & & 48.18 & [3.24-716.2] & 0.005 \\
\hline
\end{tabular}

HR, Hazard Ratio; Ref. indicates as reference level; IFN, interferon; LNR, Lymph Node Ratio

In another study, Xing et al., (2009) assessed 2,348 patients with a primary melanoma site in the head and neck and extremities undergoing lymph node dissection. Contrary to the other studies in this regard, Xing et al., (2009) defined a different cutoff point for each lymph node region (head: 0.07 , axillary region: 0.13 , inguinal region: 0.18 ), so that the survival rate increased by $50 \%$ in the patients with lower LNR in the lymph node region compared to the cutoff point. Moreover, the authors believed that 15,8 , and 6 lymph nodes had to be removed from the neck, axillary region, and inguinal region, respectively versus one metastatic lymph node. The risk of remaining metastatic lymph nodes increases in the patients who receive less lymph node dissection than the determined number. In the mentioned study, selection of different cutoff points for various lymph node regions could be due to the diverse skills and approaches adopted by the surgeons.

In a research conducted by Burger et al., (2012) on 168 patients, LNR was considered an independent prognostic factor for survival, and LNR was divided into three categories based on the study by Rossi et al., (2008) According to the results, the mortality rate was four times higher in the patients with the LNR of $>0.25$ compared to the subjects with the LNR of $<0.1$. Moreover, AIC was applied by Burger et al., (2012) to demonstrate the higher efficacy of LNR as a model to assess survival compared to the $\mathrm{N}$ model.

In this regard, Mocellin et al., (2011) examined 8,177 patients undergoing surgery to remove a minimum of five lymph nodes. Contrary to similar studies, Mocellin et al., (2011) assessed LNR as a continuous variable in association with survival, with the LNR maintaining its prognostic effect, so that each percent of increased LNR was associated with a $2 \%$ increase in the risk of mortality.

In the present study, LNR was evaluated as both a continuous and a categorized variable. According to our findings, LNR could be an independent factor for the prediction of OS. Various cutoff points have been suggested in previous studies, and we used a new cutoff value in our research. The LNR model with the cutoff point used in the present study was observed to be more 
accurate in the estimation of the survival rate compared to the AJCC N stage model. The main limitations of the present study were the retrospective design and smaller sample size compared to the similar studies in this regard.

In conclusion, the results of the present study indicated that LNR could be used as an independent prognostic factor in all patients regardless of undergoing CLND. In addition, the patients with incomplete dissection who are prone to underestimation could be evaluated with LNR.

\section{References}

Balch CM, Gershenwald JE, Soong SJ, et al (2010). Multivariate analysis of prognostic factors among 2,313 patients with stage III melanoma: comparison of nodal micrometastases versus macrometastases. J Clin Oncol, 28, 2452-9.

Berger AC, Fierro M, Kairys JC, et al (2012). Lymph node ratio is an important and independent prognostic factor for patients with stage III melanoma. J Surg Oncol, 105, 15-20.

Berger AC, Sigurdson ER, LeVoyer T, et al (2005). Colon cancer survival is associated with decreasing ratio of metastatic to examined lymph nodes. J Clin Oncol, 23, 8706-12.

Bhamidipati CM, Stukenborg GJ, Thomas CJ, et al (2012). Pathologic lymph node ratio is a predictor of survival in esophageal cancer. Ann Thorac Surg, 94, 1643-51.

Bilimoria KY, Balch CM, Bentrem DJ, et al (2008). Complete lymph node dissection for sentinel node-positive melanoma: assessment of practice patterns in the United States. Ann Surg Oncol, 15, 1566-76.

Coit DG, Thompson JA, Algazi A, et al (2016). Melanoma, version 2.2016, NCCN clinical practice guidelines in oncology. J Natl Compr Canc Netw, 14, 450-73.

Dummer R, Hauschild A, Lindenblatt N, et al (2015). Cutaneous melanoma: ESMO clinical practice guidelines for diagnosis, treatment and follow-up. Ann Oncol, 26, 126-32.

Eggermont AM, Suciu S, Santinami M, et al (2008). Adjuvant therapy with pegylated interferon alfa- $2 \mathrm{~b}$ versus observation alone in resected stage III melanoma: final results of EORTC 18991, a randomised phase III trial. Lancet, 372, 117-26.

Farid SG, Falk GA, Joyce D, et al (2014). Prognostic value of the lymph node ratio after resection of periampullary carcinomas. HPB (Oxford), 16, 582-91.

Grotz TE, Huebner M, Pockaj BA, et al (2013). Limitations of lymph node ratio, evidence-based benchmarks, and the importance of a thorough lymph node dissection in melanoma. Ann Surg Oncol, 20, 4370-7.

Mocellin S, Pasquali S, Rossi CR, et al (2011). Validation of the prognostic value of lymph node ratio in patients with cutaneous melanoma: a population-based study of 8,177 cases. Surgery, 150, 83-90.

Rossi CR, Mocellin S, Pasquali S, et al (2008). N-ratio: a novel independent prognostic factor for patients with stage-III cutaneous melanoma. Ann Surg Oncol, 15, 310-5.

Sandro P, Andrea M, Nicola M, et al (2015). Lymph-node ratio in patients with cutaneous melanoma: A multi-institution prognostic study. Ann Surg Oncol, 22, 2127-34.

Sano D, Yabuki K, Takahashi H, et al (2018). Lymph node ratio as a prognostic factor for survival in patients with head and neck squamous cell carcinoma. Auris Nasus Larynx, 45, 846-53.

Spillane AJ, Winstanley J, Thompson JF (2009). Lymph node ratio in melanoma: A marker of variation in surgical quality? Cancer, 115, 2384-7.

Truong PT, Vinh-Hung V, Cserni G, et al (2008). The number of positive nodes and the ratio of positive to excised nodes are significant predictors of survival in women with
Lymph Node Ratio on Survival in Patients with Melanoma

micrometastatic node-positive breast cancer. Eur J Cancer, 44, 1670-7.

Wang J, Hassett JM, Dayton MT, et al (2008). Lymph node ratio: role in the staging of node-positive colon cancer. Ann Surg Oncol, 15, 1600-8.

Wong SL, Faries MB, Kennedy EB, et al (2018). Sentinel lymph node biopsy and management of regional lymph nodes in melanoma: American society of clinical oncology and society of surgical oncology clinical practice guideline update. J Clin Oncol, 36, 399-413.

Xing Y, Badgwell BD, Ross MI, et al (2009). Lymph node ratio predicts disease-specific survival in melanoma patients. Cancer, 115, 2505-13.

Yamamoto Y, Ikoma H, Morimura R, et al (2014). The clinical impact of the lymph node ratio as a prognostic factor after resection of pancreatic cancer. Anticancer Res, 34, 2389-94.

Zhan HX, Xu JW, Wang L, et al (2015). Lymph node ratio is an independent prognostic factor for patients after resection of pancreatic cancer. World J Surg Oncol, 13, 105.

Zhang H, Wang W, Diao D, et al (2013). Ratio of metastatic to examined lymph nodes, a helpful staging system and independent prognostic factor of esophagogastric junction cancer. PLoS One, 8, e73238.

Zhou D, Ye M, Bai Y, et al (2015). Prognostic value of lymph node ratio in survival of patients with locally advanced rectal cancer. Can J Surg, 58, 237-44.

Zhou Y, Zhang J, Cao S, et al (2013). The evaluation of metastatic lymph node ratio staging system in gastric cancer. Gastric Cancer, 16, 309-17.

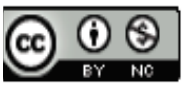

This work is licensed under a Creative Commons AttributionNon Commercial 4.0 International License. 